\title{
THE REFLECTIVE FUNCTION REPRESENTED BY THREE EXPONENTIAL MATRIXES
}

\author{
ZhengXin ZHOU
}

\begin{abstract}
In this article, we discuss the reflective function which can be represented by three exponential matrixes and apply the results to studying the existence of periodic solutions of these systems. The obtained conclusions extend and improve the foregoing results.
\end{abstract}

\section{Introduction}

As we know, to study the property of the solutions of differential system

$$
x^{\prime}=X(t, x) .
$$

is very important not only for the theory of ordinary differential equation but also for practical reasons. If $X(t+2 \omega, x)=X(t, x)$ ( $\omega$ is a positive constant), to study the solutions' behavior of (1), we could use, as introduced in [1], the Poincaré mapping. But it is very difficult to find the Poincaré mapping for many systems which cannot be integrated in quadratures. In the 1980's the Russian mathematician Mironenko [3] first established the theory of reflective functions (RF). Since then a quite new method to study (1) has been found.

In the present section, we introduce the concept of the reflective function, which will be used throughout the rest of this article.

Now consider the system (1) with a continuously differentiable right-hand side and with a general solution $\psi\left(t ; t_{0}, x_{0}\right)$. For each such system, the reflective function (RF) of (1) is defined as $F(t, x):=\psi(-t ; t, x)$. Then for any solution $x(t)$ of (1), we have $F(t, x(t))=x(-t)$. If system (1) is $2 \omega$-periodic with respect to $t$, and $F$ is its $\mathbf{R F}$, then $F(-\omega, x)=\psi(\omega ;-\omega, x)$ is the Poincaré $[1,3]$ mapping of $(1)$ over the period $[-\omega, \omega]$. So, for any solution $x(t)$ of $(1)$ defined on $[-\omega, \omega]$, it will be $2 \omega$-periodic if and only if $x(-\omega)$ is a fixed point of the Poincaré mapping $T(x)=F(-\omega, x)$.

Received August 14, 2008.

2000 Mathematics Subject Classification. 34A12.

Key words and phrases. reflecting function, periodic system, asymptotic behavior.

This Work supported by the NSF of Jiangsu Province of China BK2007075 and 08KJB110013 and the NSF of China under Grant 60774073. 
A function $F(t, x)$ is a reflective function of system (1) if and only if it is a solution of the partial differential equation

$$
F_{t}^{\prime}+F_{x}^{\prime} X(t, x)+X(-t, F)=0
$$

with the initial condition $F(0, x)=x$. It implies that for non-integral periodic systems we also can find out its Poincaré mapping. If, for example, $X(t, x)+$ $X(-t, x) \equiv 0$, then $T(x)=x$.

If $F(t, x)$ is the $\mathbf{R F}$ of (1), then it is also the $\mathbf{R F}$ of the system

$$
\dot{x}=X(t, x)+F_{x}^{\prime-1} R(t, x)-R(-t, F(t, x)),
$$

where $R(t, x)$ is an arbitrary vector function such that the solutions of the above systems are uniquely determined by their initial conditions. Therefore, all these $2 \omega$-periodic systems have a common Poincaré mapping over the period $[-\omega, \omega]$, and the behavior of the periodic solutions of these systems are the same.

See the articles $[3,4,5,6,7,8,9]$ which are also devoted to investigations of qualitative behavior of solutions of differential systems with help of reflecting function.

Let system (1) be linear, i.e.,

$$
x^{\prime}=P(t) x, t \in \mathbb{R}, x \in \mathbb{R}^{n},
$$

where $P(t)$ is a continuously differentiable $n \times n$ matrix function in $\mathbb{R}$. And suppose that $\Phi(t)$ is the fundamental matrix of solutions of (3). Then the general solution of $(3)$ is $x=\varphi\left(t ; t_{0}, x_{0}\right)=\Phi(t) \Phi^{-1}(t) x_{0}$. Therefore, the $\mathbf{R F}$ of (3) is $F(t, x)=F(t) x$, where $F(t)=\Phi(-t) \Phi^{-1}(t)$. This matrix $F(t)$ is referred to as a reflective matrix $(\mathbf{R M})$ of system (3).

$\mathbf{R M}$ of any system satisfies the relations $F(-t) F(t) \equiv F(0)=E$, where $E$ is the $n \times n$ unit matrix. Differentiable matrix $F(t)$ is a $\mathbf{R M}$ of system (3) if and only if it is a solution of the system (basic relation)

$$
F^{\prime}(t)+F(t) P(t)+P(-t) F(t)=0
$$

with the initial condition $F(0)=E$. Any linear system with reflecting matrix $F(t)$ can be reduced in the form

$$
x^{\prime}=\left[-\frac{1}{2} F(-t) F^{\prime}(t)+F(-t) R(t)-R(-t) F(t)\right] x,
$$

where $R(t)$ is an arbitrary continuous real matrix.

If matrix $P(t)$ is $2 \omega$-periodic and $F(t)$ is $\mathbf{R M}$ of system (3), then for this system the matrix $F(-\omega)$ is the monodromy matrix on the interval $[-\omega, \omega]$. Thus solutions $\mu_{i}(i=1,2, \ldots, n)$ of the equation $\operatorname{det}(F(-\omega)-\mu E)=0$ are the multiplicators of system (3)

As we known, when $P(t+2 \omega)=P(t)$, the fundamental matrix $\Phi(t)$ of (3) can be represented in the form $\Phi(t)=\Psi(t) e^{B t}$, where $\Psi(t)$ is a continuous periodic $n \times n$ matrix and det $\Psi(t) \neq 0, B$ is a constant $n \times n$ matrix. RM of such systems is $F(t)=\Phi(-t) \Phi^{-1}(t)=\Psi(-t) e^{2 B t} \Psi^{-1}(t)$. With this in mind we suppose that $\mathbf{R M}$ of system (3) is given by $F(t)=e^{A(-t)} e^{2 B t} e^{-A(t)}$, where $A(t)$ is a $n \times n$ 
matrix function, $B$ is a constant $n \times n$ matrix. In the papers of Musafirov $[5,6]$, the forms of RM $F(t)=e^{A t} e^{2 B t} e^{A t}$ and $F(t)=e^{\alpha(t) A} e^{\beta(t) B} e^{-\alpha(-t) A}$ have been discussed. In the following, we will discuss when the RM of system (3) has the more general form of $F(t)=e^{A(-t)} e^{2 B t} e^{-A(t)}$. It will develop the results of Musafirov [5, 6].

\section{Main results}

Theorem 1. $F(t)=e^{A(-t)} e^{2 B t} e^{-A(t)}$ is the $\mathbf{R M}$ of system (3), if and only if

$$
F(t) D(t)+D(-t) F(t)=0
$$

where $D(t)=e^{A(t)} B e^{-A(t)}+P(t)-A^{\prime}(t)$.

Proof. By the foregoing introduction, we see that $F(t)=e^{A(-t)} e^{2 B t} e^{-A(t)}$ is the RM of (3), if and only if,

$$
F^{\prime}(t)+F(t) P(t)+P(-t) F(t)=0,
$$

simple computing, we get

$F(t)\left(e^{A(t)} B e^{-A(t)}+P(t)-A^{\prime}(t)\right)+\left(e^{A(-t)} B e^{-A(-t)}+P(-t)-A^{\prime}(-t)\right) F(t)=0$,

i.e.,

$$
F(t) D(t)+D(-t) F(t)=0,
$$

where $D(t)=e^{A(t)} B e^{-A(t)}+P(t)-A^{\prime}(t)$.

Putting $t=0$, we get $D(0)=0$ and matrix $B$.

Theorem 2. $F(t)=e^{A(-t)} e^{2 B t} e^{-A(t)}$ is the $\mathbf{R M}$ of system (3) if and only if

$$
P(t)=A^{\prime}(t)-e^{A(t)} B e^{-A(t)}+e^{A(t)} e^{-B t} N(t) e^{B t} e^{-A(t)},
$$

where $N(t)$ ia an odd continuously differentiable $n \times n$ matrix function, $B$ is the same as in the relation (5).

Proof. According to Theorem 1, we know $F(t)=e^{A(-t)} e^{2 B t} e^{-A(t)}$ is the RM of system (3), if and only if, $F(t) D(t)+D(-t) F(t)=0$, i.e.,

$$
e^{A(-t)} e^{2 B t} e^{-A(t)} D(t)+D(-t) e^{A(-t)} e^{2 B t} e^{-A(t)}=0,
$$

which implies

$$
e^{B t} e^{-A(t)} D(t) e^{A(t)} e^{-B t}+e^{-B t} e^{-A(-t)} D(-t) e^{A(-t)} e^{B t}=0 .
$$

Putting $N(t)=e^{B t} e^{-A(t)} D(t) e^{A(t)} e^{-B t}$, then $N(t)+N(-t)=0$, and $D=$ $e^{A(t)} e^{-B t} N(t) e^{B t} e^{-A(t)}$. Thus, $P(t)$ can be expressed by relation (6). 
Corollary 1. If $P(t)=A^{\prime}(t)-e^{A(t)} B e^{-A(t)}+\gamma(t) E$, then

$$
F(t)=e^{A(-t)} e^{2 B t} e^{-A(t)}
$$

is the $\mathbf{R M}$ of system (3). Here $\gamma(t)$ is an arbitrary continuously differentiable scalar function, $E$ is an identity $n \times n$ matrix, $B$ is the same as in the relation (5).

Proof. In Theorem 2, taking $N(t)=\gamma(t) E$, this implies the present result.

Corollary 2. If $P(t)=\left(\alpha^{\prime}(t)+\gamma(t)-\alpha^{\prime}(0)\right) E-P(0)$, then

$$
F(t)=e^{\alpha(-t)-\alpha(t)} e^{2 B t}
$$

is the $\mathbf{R M}$ of system (3). Here $B=\alpha^{\prime}(0) E+P(0), E$ is an identity matrix, $\alpha(t), \gamma(t)$ are scalar continuously differentiable functions, odd functions.

Proof. In Corollary 1 taking $A(t)=\alpha(t) E$, which yields the present result.

Theorem 3. Suppose that $P(t+2 \omega)=P(t)$ and $F(t)=e^{A(-t)} e^{2 B t} e^{-A(t)}$ is the $\mathbf{R M}$ of system (3), then

1) The Poincaré mapping of (3) is $T(x)=F(-\omega) x=e^{A(\omega)} e^{-2 B \omega} e^{-A(-\omega)} x$.

2) The solution $x=\phi\left(t,-\omega, x_{0}\right)$ of (3) is $2 \omega$-periodic if and only if $F(-\omega) x_{0}=$ $x_{0}$.

3) If $x(t)$ is an arbitrary solution of $(3)$, then $y(t)=e^{B t} e^{-A(t)} x(t)$ is an even function.

Proof. The conclusions 1 and 2 can be reduced by the properties of reflective function.

Now we shall prove the conclusion 3 is true. In factor, because $F(t)=$ $e^{A(-t)} e^{2 B t} e^{-A(t)}$ is the RM of $(3)$, then $e^{A(-t)} e^{2 B t} e^{-A(t)} x(t)=x(-t)$, i.e., $e^{2 B t} e^{-A(t)} x(t)=e^{-A(-t)} x(-t)$, and so $e^{B t} e^{-A(t)} x(t)=e^{-B t} e^{-A(-t)} x(-t)$, that is $y(t)=y(-t)$.

Example 1. Differential system

$$
x^{\prime}=P(t) x=\left(\begin{array}{cc}
-\sin t-1+\frac{1}{2} \operatorname{sh}(2 \sin t) & \cos t-\operatorname{ch}^{2}(\sin t) \\
\cos t+\operatorname{sh}^{2}(\sin t) & -\sin t-1-\frac{1}{2} \operatorname{sh}(2 \sin t)
\end{array}\right) x
$$

has RM $F(t)=e^{A(-t)} e^{2 B t} e^{-A(t)}$, in which

$$
A(t)=\left(\begin{array}{cc}
\cos t & \sin t \\
\sin t & \cos t
\end{array}\right), B=\left(\begin{array}{ll}
2 & 2 \\
0 & 2
\end{array}\right) .
$$

It is easy to check that $P(t)=A^{\prime}(t)-e^{A(t)} B e^{-A(t)}$ is true. Since this system is $2 \pi$-periodic, then the Poincaré mapping is $T(x, y)=F(-\pi) x$. In view of $\operatorname{det}(F(-\pi)-E)=\left(e^{-2 \pi}-1\right)^{2} \neq 0$, thus, the present system exists unique $2 \pi$-periodic solution which is asymptotically stable. 
Theorem 4. Suppose that

$$
\begin{aligned}
& P(t)=A^{\prime}(t)-e^{A(t)} B e^{-A(t)}+\sum_{k=1}^{m} \gamma_{k}(t) M^{k}(t), \\
& M^{\prime}=P(t) M(t)-M(t) P(t)+\sum_{k=1}^{m} \beta_{k}(t) M^{k}(t) .
\end{aligned}
$$

Then $F(t)=e^{A(-t)} e^{2 B t} e^{-A(t)}$ is the $\mathbf{R M}$ of system $(3)$. Here $\gamma_{k}(t), \beta_{k}(t), k=$ $1,2, \ldots, m$ are odd continuously differentiable scalar functions, $M(t)$ is a $n \times n$ matrix function, $B=e^{-A(0)}\left(A^{\prime}(0)-P(0)\right) e^{A(0)}$.

Proof. By Theorem 1, to prove that $F(t)=e^{A(-t)} e^{2 B t} e^{-A(t)}$ is the RM of system (3), only need to check out the relation (4) to be hold. Using the hypothesis of the present theorem, we have $D(t)=\sum_{k=1}^{m} \gamma_{k}(t) M^{k}(t)$. Thus

$$
\begin{aligned}
& F(t) D(t)+D(-t) F(t) \\
= & \sum_{k=1}^{m} \gamma_{k}(t)\left(F(t) M^{k}(t)-M^{k}(-t) F(t)\right) \\
= & \sum_{k=1}^{m} \gamma_{k}(t) \sum_{i+j=k-1} M^{i}(-t)(F(t) M(t)-M(-t) F(t)) M^{j}(t) .
\end{aligned}
$$

Let denote $U=F(t) M(t)-M(-t) F(t)$. Then $U(0)=0$ and

$$
F(t) D(t)+D(-t) F(t))=\sum_{k=1}^{m} \gamma_{k}(t) \sum_{i+j=k-1} M^{i}(-t) U M^{j}(t)
$$

Since $F(t)=e^{A(-t)} e^{2 B t} e^{-A(t)}$, so $F^{\prime}(t)=F(t)\left(S-A^{\prime}\right)+\left(\bar{S}-\bar{A}^{\prime}\right) F(t)$, in which, $S=S(t)=e^{A(t)} B e^{-A(t)}, \bar{S}=S(-t), A=A(t), \bar{A}=A(-t)$. Therefore,

$$
\begin{aligned}
U^{\prime}= & F(t)\left(M^{\prime}(t)+S M-A^{\prime} M\right)-\left(\bar{M} \bar{A}^{\prime}+\bar{M} \bar{S}+\bar{M}^{\prime}\right) F(t) \\
& +\left(\bar{A}^{\prime}+\bar{S}\right) F M-\bar{M} F\left(S-A^{\prime}\right) \\
= & F\left(P M-M P+\left(S-A^{\prime}\right) M+\sum_{k=1}^{m} \beta_{k}(t) M^{k}\right) \\
& +\left(\bar{P} \bar{M}-\bar{M} \bar{P}-\bar{M}\left(\bar{A}^{\prime}+\bar{S}\right)+\sum_{k=1}^{m} \beta_{k}(t) M^{k}\right) F \\
= & -\bar{P} U-U P+\sum_{k=1}^{m} \beta_{k}(t) \sum_{i+j=k-1} \bar{M}^{i} U M^{j} \\
& +\sum_{k=1}^{m} \gamma_{k}(t) \sum_{i+j=k-1} \bar{M}^{i} U M^{j+1}-\sum_{k=1}^{m} \gamma_{k} \sum_{i+j=k-1} \bar{M}^{i+1} U M^{j} .
\end{aligned}
$$


By the uniqueness of solution of the initial problem of the linear partial differential equation, this implies $U(t) \equiv 0$. Thus,

$$
F(t) D(t)+D(-t) F(t)=\sum_{k=1}^{m} \gamma_{k}(t) \sum_{i+j=k-1} \bar{M}^{i} U M^{j}=0,
$$

By Theorem 1, $F(t)=e^{A(-t)} e^{2 B t} e^{-A(t)}$ is the $\mathbf{R M}$ of system 3 .

Theorem 5. Suppose that $F(t)=e^{A(-t)} e^{2 B t} e^{-A(t)}$ is the RM of system (3). Then

$$
\begin{aligned}
& P^{\prime \prime}(0)-A^{(3)}(0)+A^{\prime \prime}(0) A^{\prime}(0)-A^{\prime}(0) A^{\prime \prime}(0)+2 A^{\prime}(0) P(0) A^{\prime}(0) \\
& +P(0)\left(3 A^{\prime \prime}(0)-A^{\prime 2}(0)-2 P^{\prime}(0)+2 A^{\prime}(0) P(0)-2 P(0) A^{\prime}(0)\right) \\
& +\left(2 P^{\prime}(0)-3 A^{\prime \prime}(0)-{A^{\prime}}^{2}(0)-2 A^{\prime}(0) P(0)+2 P(0) A^{\prime}(0)\right) P(0)=0,
\end{aligned}
$$

and

$$
B=e^{-A(0)}\left(A^{\prime}(0)-P(0)\right) e^{A(0)} .
$$

Proof. By Theorem 1, we have $F(t) D(t)+D(-t) F(t)=0$. Setting $t=0$, we obtain matrix $B$. Twice differentiating relation $F(t) D(t)+D(-t) F(t)=0$, and setting $t=0$, we get

$$
D^{\prime \prime}(0)+2 D^{\prime}(0) P(0)-2 P(0) D^{\prime}(0)=0 .
$$

Since $D(t)=e^{A(t)} B e^{-A(t)}+P(t)-A^{\prime}(t)$, then $D^{\prime}(0)=P^{\prime}(0)-A^{\prime \prime}(0)-$ $A^{\prime}(0) P(0)+P(0) A^{\prime}(0), D^{\prime \prime}(0)=P^{\prime \prime}(0)-A^{\prime \prime \prime}(0)+A^{\prime \prime}(0) A^{\prime}(0)-A^{\prime}(0) A^{\prime \prime}(0)-$ $\left(A^{\prime \prime}\left(0+A^{\prime 2}(0)\right)\right) P(0)+P(0)\left(A^{\prime \prime}(0)-A^{\prime 2}(0)\right)+2 A^{\prime}(0) P(0) A^{\prime}(0)$. Substituting these into $(8)$, it implies the identity $(7)$.

Remark 1. Suppose that $F(t)=e^{A(-t)} e^{2 B t} e^{-A(t)}$ is the $\mathbf{R M}$ of system (3) and $A(t)+A(-t)=0$. Then

$$
\begin{aligned}
& P^{\prime \prime}(0)-A^{(3)}(0)+2 A^{\prime}(0) P(0) A^{\prime}(0)+P(0)\left(-A^{\prime 2}(0)\right. \\
& \left.-2 P^{\prime}(0)+2 A^{\prime}(0) P(0)-2 P(0) A^{\prime}(0)\right) \\
& +\left(2 P^{\prime}(0)-A^{\prime 2}(0)-2 A^{\prime}(0) P(0)+2 P(0) A^{\prime}(0)\right) P(0)=0,
\end{aligned}
$$

and

$$
B=A^{\prime}(0)-P(0) .
$$

Remark 2. Suppose that $F(t)=e^{A(-t)} e^{2 B t} e^{-A(t)}$ is the $\mathbf{R M}$ of system (3) and $A(t)=A t$. Then

$$
\begin{aligned}
& P^{\prime \prime}(0)+2 A P(0) A+P(0)\left(-A^{2}-2 P^{\prime}(0)+2 A P(0)-2 P(0) A\right) \\
& +\left(2 P^{\prime}(0)-A^{2}-2 A P(0)+2 P(0) A\right) P(0)=0
\end{aligned}
$$

and

$$
B=A-P(0)
$$


Remark 3. Suppose that $F(t)=e^{A(-t)} e^{2 B t} e^{-A(t)}$ is the $\mathbf{R M}$ of system (3) and $A(t)=A(-t)$. Then

$$
P^{\prime \prime}(0)+P(0)\left(3 A^{\prime \prime}(0)-2 P^{\prime}(0)\right)+\left(2 P^{\prime}(0)-3 A^{\prime \prime}(0)\right) P(0)=0,
$$

and

$$
B=-e^{-A(0)} P(0) e^{A(0)} .
$$

Theorem 6. Suppose that $F(t)=e^{-A(t)} e^{2 B t} e^{-A(t)}$ is the RM of system (3) and $A(t)+A(-t)=0$. Then there exists an odd $n \times n$ function matrix $N(t)$ such that

$$
\begin{aligned}
& e^{A(t)}(P(t)-P(-t)) e^{A(t)} \\
= & B e^{2 A(t)}-e^{2 A(t)} B+e^{2 A(t)} e^{-B t} N(t) e^{B t}+e^{B t} N(t) e^{-B t} e^{2 A(t)},
\end{aligned}
$$

where $B=A^{\prime}(0)-P(0), A^{\prime}(0) P(0)-P(0) A^{\prime}(0)=P^{\prime}(0)-N^{\prime}(0)$.

Proof. Using Theorem 2, there exists an odd $n \times n$ function matrix $N(t)$ such that

$$
P(t)=A^{\prime}(t)-e^{A(t)} B e^{-A(t)}+e^{A(t)} e^{-B t} N(t) e^{B t} e^{-A(t)},
$$

SO

$$
P(-t)=A^{\prime}(t)-e^{-A(t)} B e^{A(t)}-e^{-A(t)} e^{B t} N(t) e^{-B t} e^{A(t)},
$$

(10)-(11) yields the identity (9).

Since $A(t)+A(-t)=0$, then $A(0)=A^{\prime \prime}(0)=0$. Differentiating (10) and setting $t=0$ we get $A^{\prime}(0) P(0)-P(0) A^{\prime}(0)=P^{\prime}(0)-N^{\prime}(0)$.

Remark 4. Solving equation $A^{\prime}(0) P(0)-P(0) A^{\prime}(0)=P^{\prime}(0)-N^{\prime}(0)$ we obtain $A^{\prime}(0)$, solving

$$
\begin{aligned}
& e^{A(t)}(P(t)-P(-t)) e^{A(t)} \\
= & B e^{2 A(t)}-e^{2 A(t)} B+e^{2 A(t)} e^{-B t} N(t) e^{B t}+e^{B t} N(t) e^{-B t} e^{2 A(t)},
\end{aligned}
$$

we obtain $e^{A(t)}$. If $e^{A(t)}$ satisfies

$$
\left(e^{A(t)}\right)^{\prime}=P(t) e^{A(t)}+e^{A(t)} B-e^{A(t)} e^{-B t} N(t) e^{B t},
$$

in which $B=A^{\prime}(0)-P(0)$, then $F(t)=e^{-A(t)} e^{2 B t} e^{-A(t)}$ is the RM of system (3).

In the similar way we have

Theorem 7. Suppose that $F(t)=e^{A(t)} e^{2 B t} e^{-A(t)}$ is the RM of system (3) and $A(t)=A(-t)$. Then there exists an odd $n \times n$ function matrix $N(t)$ such that

(12) $(P(t)+P(-t)) e^{A(t)}+2 e^{A(t)} B=e^{A(t)}\left(e^{-B t} N(t) e^{B t}-e^{B t} N(t) e^{-B t}\right)$, where $B=-e^{-A(0)} P(0) e^{A(0)}, A^{\prime \prime}(0)+e^{A(0)} N^{\prime}(0) e^{-A(0)}=P^{\prime}(0), P^{\prime \prime}(0)=$ $\left(3 A^{\prime \prime}(0)-2 P^{\prime}(0)\right) P(0)-P(0)\left(3 A^{\prime \prime}(0)-2 P^{\prime}(0)\right)$. 
Remark 5. Solving equation

$$
(P(t)+P(-t)) e^{A(t)}+2 e^{A(t)} B=e^{A(t)}\left(e^{-B t} N(t) e^{B t}-e^{B t} N(t) e^{-B t}\right)
$$

we obtain $e^{A(t)}$, then we get $B=-e^{-A(0)} P(0) e^{A(0)}$. If $e^{A(t)}$ satisfies

$$
\left(e^{A(t)}\right)^{\prime}=P(t) e^{A(t)}+e^{A(t)} B-e^{A(t)} e^{-B t} N(t) e^{B t},
$$

then $F(t)=e^{A(t)} e^{2 B t} e^{-A(t)}$ is the RM of system (3).

Using the Theorem 1 in [4, p.1397], we obtain

Theorem 8. Suppose that the vector function $\Delta(t, x)$ satisfies identity

$$
\Delta^{\prime}(t, x)_{t}+\Delta^{\prime}(t, x)_{x} P(t) x=P(t) \Delta(t, x)
$$

and $F(t) x=e^{A(t)} e^{2 B t} e^{-A(t)} x$ is the $\mathbf{R F}$ of system $(3), \alpha(t)$ is an arbitrary odd continuously differentiable scalar function. Then $F(t) x=e^{A(t)} e^{2 B t} e^{-A(t)} x$ is also $\mathbf{R F}$ of nonlinear differential system

$$
x=P(t) x+\alpha(t) \Delta(t, x) .
$$

Besides this, if system (13) is $2 \omega$-periodic, then the properties of periodic solution of (13) are the same as the periodic solutions of system (3).

Consider the nonlinear differential system with small parameter $\varepsilon$

$$
x^{\prime}=F(t, x, \varepsilon), t \in \mathbb{R}, x \in D \subset \mathbb{R}^{n},
$$

where $F$ is a continuous $2 \omega$-periodic vector function for all $t$, and also continuously differentiable with respect to components of a vector $x, \varepsilon$ is small. Let $x=\varphi(t)$ be a $2 \omega$-periodic solution of the system (14) when $\varepsilon=0$.

Theorem 9. Let $F(t)=e^{A(t)} e^{2 B t} e^{-A(t)}$ be the RM of system (3) with matrix

$$
P(t)=\frac{\partial F}{\partial x}(t, \varphi(t), 0) .
$$

If there is no unit among solutions $\mu_{i}$ of equation

$$
\operatorname{det}\left(e^{A(-\omega)} e^{-2 B \omega} e^{-A(-\omega)}-\mu E\right)=0,
$$

then system (14) with sufficiently small $|\varepsilon|$ has the unique $2 \omega$-periodic solution $x=x(t, \varepsilon)$ with an initial point $x(0, \varepsilon)$ close to $\varphi(t)$. Besides, $x(t, \varepsilon)$ is a continuous function with respect to $(t, \varepsilon)$, and $x(t, 0)=\varphi(t)$. If, moreover, $F$ is continuously differentiable with respect to $\varepsilon$, then $x(t, \varepsilon)$ is also continuously differentiable.

Proof. Since multiplicators $\mu_{i}$ for $2 \omega$-periodic linear system with the RM

$$
F(t)=e^{A(t)} e^{2 B t} e^{-A(t)}
$$

are solutions of equation

$$
\operatorname{det}\left(e^{A(-\omega)} e^{-2 B \omega} e^{-A(-\omega)}-\mu E\right)=0,
$$

therefore validity of this theorem follows from the Theorem 2.3 in [2, p.488]. 


\section{References}

[1] V. I. Arnol'd, Ordinary Differential Equation, Science Press, Moscow, 1971.

[2] P. Hartman, Ordinary Differential Equations, Moscow, 1970.

[3] V. I. Mironenko, Reflecting Function and Periodic Solutions of Differential Equations, Univ. Press, Minsk, 1986.

[4] V. V. Mironenko, Time symmetry preserving perturbations of differential systems, Differ. Equ. 40 (2004), no. 10, 1395-1403.

[5] E. V. Musafirov, Differential systems, the mapping over period for which is represented by a product of three exponential matrixes, J. Math. Anal. Appl. 329 (2007), no. 1, 647-654.

[6] - On differential systems whose reflection matrix is the product of matrix exponentials, Vestsi Nats. Akad. Navuk Belarusi Ser. Fiz.-Mat. Navuk 2002 (2002), no. 1, 44-50, 126.

[7] P. P. Verecovitch, Nonautonomous two-dimensional quadratic systems that are equivalent to linear systems, Differ. Equ. 34 (1998), no. 10, 1421-1424.

[8] Z. Zhengxin, On the reflective function of polynomial differential system, J. Math. Anal. Appl. 278 (2003), no. 1, 18-26.

[9] Z. Zhengxin and Y. Yuexin, On the reflective function for two-dimensional quadratic differential systems, Math. Appl. (Wuhan) 15 (2002), no. 4, 85-91.

Department of Mathematics

YANGZHOU UNIVERSITY

JiAngsu 225002, P. R. ChinA

E-mail address: zhengxinzhou@hotmail.com 